\title{
Status and results of merging different strains of Brown cattle in Europe
}

\author{
G. AVERDUNK \\ Bayerische Landesanstalt $f$. Tierzucht, D 8011 Grub b München, \\ B.R.D.
}

In the European Braunvieh-populations since 1965 American Brown-Swiss bulls were used to increase genetic variation and milk production. While in the beginning of this period not all countries in Europe were involved, the situation now has changed: All countries are using combinations of American and European genotypes and the majority of test bulls has 50 and 75 p. 100 BS-genes. At present there are no indications of a complete replacement of European genotypes, but a combination is aimed at.

While the early analysis of data showed large breed differences with negative heterosis and recombination loss, newer data showed an advantage in milk yield, fat and protein yield, while the protein content was slightly lower. The progeny of 5/8- and 3/4-BS-bulls were on average as good in milk-production as the purebred American bulls, indicating that a combination of European and American strains is successful.

Rather limited information on fattening ability and carcass-quality indicate, that the crosses are taller, have a comparable or higher rate of gain with lower dress-out-p. 100. Higher internal fat leads in connection with heavier bones to a decrease in high priced cuts and to inferior carcass grading. With regard to secondary traits, the calving intervall of progeny from $100 \mathrm{p} .100$ BS-bulls tends to be longer, but longer gestation and lower culling rates could have caused this findings. Milkability and calving ease were influenced slightly in a favourable direction.

Finally the possibilities of comparing European populations according to the IDF-proposals are discussed. Links between the European populations at present are only through the use of American bulls, most of them were progeny-tested and used in Europe in planned matings. An approach should be taken, to use this material to get hints about the base differences between the European countries. Nevertheless it should be tried to organise an exchange of semen from test bulls across countries and to get an unbiased proof on these in each of the cooperating countries.

\section{Population genetic examination on the heterosis effects in crossbreeding Hungarian Simmental cattle with Herefords 4}

\author{
F. SZABO \\ University of Agricultural Sciences Keszthely, \\ Faculty of Agricultural Sciences Keszthely, Hungary
}

We studied the heterosis effects in characteristics having fundamental influence on meat production as a result of crossbreeding Hungarian Simmental cattle with Herefords.

Evaluating a considerable number of data, $F_{1}$ population was found to be superior not only to the average of the two purebreed populations, but also to that of the better parent population in major maternal characteristics. As a result of crossing, the following results were found as a consequence of heterosis effects : 7-8 p. 100 at the age of first calving, 6-9 p. 100 in the period between two calvings, $5-8$ p. 100 at calving rate and $2-3$ p. 100 in the rate of weaned calves.

Live weight, boned meat and meat production for 1 day of life and the percentage of meat, pectoral and abdominal tallow and tallow as a result of boning in the case of $F_{1}$ population gave better results than the average of Hungarian Simmental and Hereford, but fell short of Hungarian Simmental. tage.

No heterosis effect was found in judgement scores of live animals and in killing out percen- 